\title{
EEG-Based Drowsiness Estimation for Safety Driving Using Independent Component Analysis
}

\author{
Chin-Teng Lin, Fellow, IEEE, Ruei-Cheng Wu, Sheng-Fu Liang, Wen-Hung Chao, Yu-Jie Chen, and Tzyy-Ping Jung
}

\begin{abstract}
Preventing accidents caused by drowsiness has become a major focus of active safety driving in recent years. It requires an optimal technique to continuously detect drivers' cognitive state related to abilities in perception, recognition, and vehicle control in (near-) real-time. The major challenges in developing such a system include: 1) the lack of significant index for detecting drowsiness and 2) complicated and pervasive noise interferences in a realistic and dynamic driving environment. In this paper, we develop a drowsiness-estimation system based on electroencephalogram (EEG) by combining independent component analysis (ICA), power-spectrum analysis, correlation evaluations, and linear regression model to estimate a driver's cognitive state when he/she drives a car in a virtual reality (VR)-based dynamic simulator. The driving error is defined as deviations between the center of the vehicle and the center of the cruising lane in the lane-keeping driving task. Experimental results demonstrate the feasibility of quantitatively estimating drowsiness level using ICA-based multistream EEG spectra. The proposed ICA-based method applied to power spectrum of ICA components can successfully (1) remove most of EEG artifacts, (2) suggest an optimal montage to place EEG electrodes, and estimate the driver's drowsiness fluctuation indexed by the driving performance measure. Finally, we present a benchmark study in which the accuracy of ICA-component-based alertness estimates compares favorably to scalp-EEG based.
\end{abstract}

Index Terms-Correlation coefficient, drowsiness, electroencephalogram, independent component analysis (ICA), linear regression model, power spectrum, virtual reality (VR).

\section{INTRODUCTION}

$\mathbf{R}$ ECENTLY, driving safely has received increasing attention of the publics due to the growing number of traffic accidents. Drivers' fatigue has been implicated as a causal factor

Manuscript received February 1, 2005; revised July 24, 2005. The work of C.-T. Lin was supported in part by the Brain Research Center, University System of Taiwan, R.O.C., under Contract 93R567. The work of T.-P. Jung was supported by the Swartz Foundation. This paper was recommended by Guest Editor Y. Lian.

C.-T. Lin is with the Department of Electrical and Control Engineering/Department of Computer Science, National Chiao-Tung University, Hsin-Chu, Taiwan, R.O.C., and also with the Brain Research Center, University System of Taiwan, Taiwan, R.O.C. (e-mail: ctlin@mail.nctu.edu.tw).

R.-C. Wu and Y.-J. Chen are with the Department of Electrical and Control Engineering, National Chiao-Tung University, Hsin-Chu, Taiwan, R.O.C. (e-mail: allen.ece86g@nctu.edu.tw; blue.ece92g@nctu.edu.tw).

S.-F. Liang is with the Department of Biological Science and Technology, National Chiao-Tung University, Hsin-Chu, Taiwan, R.O.C., and also with the Brain Research Center, University System of Taiwan, Taiwan, R.O.C. (e-mail: sfliang@mail.nctu.edu.tw).

W.-H. Chao is with the Department of Biomedical Engineering, Yuanpei University of Science and Technology, Hsin-Chu, Taiwan, R.O.C., and also with the Brain Research Center, University System of Taiwan, Taiwan, R.O.C. (e-mail: wenhong@mail.yust.edu.tw).

T.-P. Jung is with the Institute for Neural Computation, University of California, San Diego, CA 92037 USA (e-mail: jung@ @scn.ucsd.edu).

Digital Object Identifier 10.1109/TCSI.2005.857555 in many accidents because of the marked decline in the drivers' abilities of perception, recognition and vehicle control abilities while sleepy. The National Highway Traffic Safety Administration (NHTSA) conservatively estimates that 100000 police-reported crashes are the direct results of driver fatigue each year [1]. This results in an estimated 1550 deaths, 71000 injuries, and $\$ 12.5$ billion in monetary losses. In 2002, the National Sleep Foundation (NSF) also reported [2] that $51 \%$ of adult drivers had driven a vehicle while feeling drowsy and $17 \%$ had actually fallen asleep. Although many governments and vehicle manufacturers try to make policies to prevent such accidents including strategies to address rates of speed, alcohol consumption; promotion of using helmets and seat belts, enhancements of vehicle structures, etc. [3], [4], the knowledge and technologies available today are still not yet enough to prevent the catastrophic incidents resulted from loss of alertness and lack of attentions on drivers intrinsically.

Driving under the influences of drowsiness will cause: 1) longer reaction time, which may lead to higher risk of crash, particularly at high speeds; 2) vigilance reduction including nonresponses or delaying responding where performance on attention-demanding tasks declines with drowsiness; 3 ) deficits in information processing, which may reduce the accuracy and correctness in decision-making [5]-[7]. Many factors can cause drowsiness or fatigue in driving including lack of sleep, long driving hours, use of sedating medications, consumption of alcohol, and some driving patterns such as driving at midnight, early morning, midafternoon hours, and especially in a monotonous driving environment [8]. Accurate and nonintrusive real-time monitoring of driver's drowsiness would be highly desirable, particularly if this measure could be further used to predict changes in driver's performance capacity.

A number of methods have been proposed to detect drowsiness in the past few years. These methods can be categorized into two major categories. One focuses on detecting physical changes during drowsiness by image processing techniques, such as average of eye-closure speed, percentage of eye-closure over time, eye tracking as quantization of drowsiness level, and driver's head movements [8]-[16]. These image-processing based methods use optical sensors or video cameras to detect eye-activity changes in drowsiness and can achieve a satisfactory recognition rate. However, these parameters might vary in different environmental situations and driving conditions, it thus might require devising different detection logics for different types of vehicles.

Other methods focused on measuring physiological changes of drivers, such as heart-rate variability (HRV), electrooculographic (EOG), or particularly, electroencephalogram (EEG), as 
a means of detecting the human cognitive states [17]-[21]. Stern et al. [22], [23] reported that the eye blink duration and blink rate typically increase while blink amplitude decreases as function of the cumulative time on tasks. Other EOG studies have found that saccade frequencies and velocities decline as time on the task increases [24], [25]. It has been known that abundant information in EEG recording covaries with drowsiness, arousal, sleep, and attention [26]. Previous psychophysiological studies show that typical sleep rhythm regulated by the circadian process can be divided into nonrapid-eye-movement (NREM) sleep and rapid-eye-movement (REM) sleep [27], [28]. NREM sleep is further subdivided into stages $1-4$. In the first part of falling into sleep (microsleep at NREM), increasing amplitudes of slow alpha waves of the EEG signals were observed with positive correlation at occipital sites (O1 and $\mathrm{O} 2)$ and negative correlation at central sites (C3 or C4) [29], [30]. Recently, Van Ordan et al. [33] compared these eye-activity based methods to EEG-based methods for alertness estimates in a compensatory visual tracking task. They showed that although these eye-activity variables are well correlated with the subject performance, those eye-activity based methods require a relatively long moving averaged window aiming to track slow or tonic changes in vigilance, whereas the EEG-based method can use a shorter moving averaged window to track second-to-second fluctuations in the subject error in a visual compensatory task [31]-[36].

While approaches based on EEG signals have the advantages in making accurate and quantitative assessment of alertness levels, relatively little information has been captured in real time until signal processing methods and computer power are fast enough to extract the relevant information from the EEG. That is, we need to explore the EEG correlates of fatigue and drowsiness, as well as to evaluate to what extent these cognitive-state related EEG activities can be efficiently incorporated into a real-time drowsiness monitoring system. One of many technical challenges of using EEG-based monitoring systems is the contamination from pervasive EEG artifacts, including muscle noise, eye activity, and blink artifacts caused by driver's hand, torso, head, and eye movement, and instrumental noises such as line noise, electronic interference, etc. Using low-pass filtering method is not sufficient to remove these contaminations. Another challenge of using the EEG-based monitoring system in operational environments is the intraand interindividual variability in EEG dynamics accompanying loss of alertness [31]-[36]. Jung et al. [31] and Lin et al. [62] have reported that the EEG correlates of fatigue and drowsiness appear stable within individual across different sessions, but somewhat variable between subjects. These results suggested the feasibility of a practical individualized system for noninvasive monitoring of the cognitive state of subjects performing auditory target detection and/or visual tracking tasks in a laboratory settings.

In this paper, we propose new methods for accurate and nonintrusive monitoring the continuous fluctuations of driver's global-level drowsiness indexed by the driving performance in near real-time in a realistic driving task. We first construct a virtual reality (VR)-based interactive driving environment consisting of a highway scene and a six degree-of-freedom

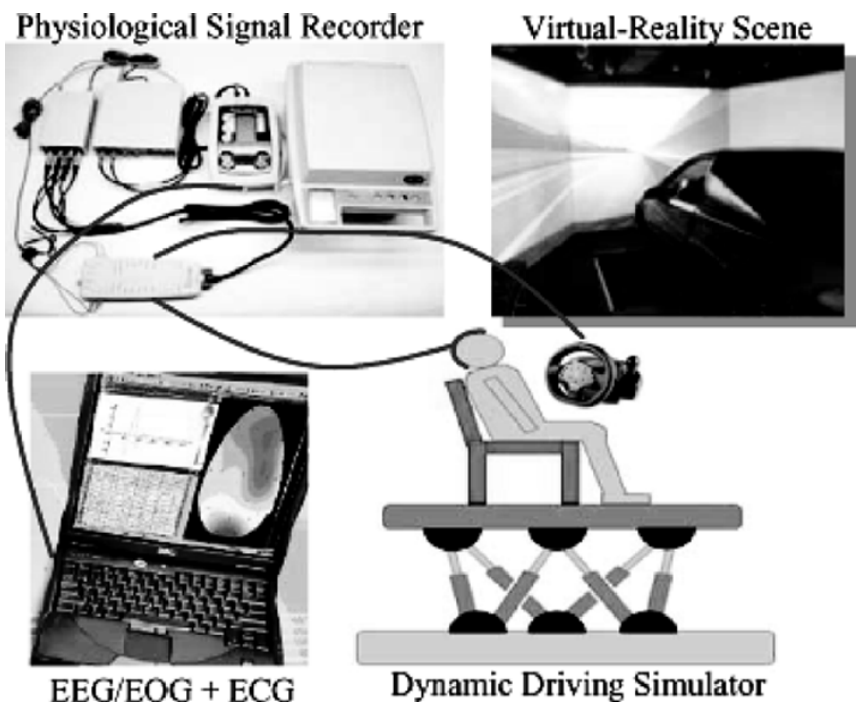

Fig. 1. The block diagram of the dynamic VR-based driving simulation environment integrated with the EEG-based physiological measurement system.

(DOF) motion platform. The VR technique allows subjects to interact directly with a virtual environment rather than passively responding to monotonic auditory and visual stimuli, and is an excellent setting for brain research to study EEG dynamics in interactive and realistic tasks. We then design a lane-keeping driving experiment to indirectly assess driver's drowsiness level by measuring second- or minute-scale fluctuations in driving errors and accompanying changes in the EEG spectra. After collecting the multistream brain potentials, independent component analysis (ICA) [37]-[52] is used to remove a wide variety of artifacts based on blind source separation and extract the representative EEG features. By correlating changes in subband log power spectra and the driving performance, we build an individualized linear regression model to assess continuously the EEG dynamics accompanying loss of alertness for each operator.

This paper is organized as follows. Section II describes the detailed descriptions of the EEG-based drowsiness experimental setup including VR-based driving environment, EEG data collection, subject's instructions, and index of drowsiness measurement. In Section III, we explore the relationship between the drowsiness level (subject driving performance) and the EEG power spectrum by combining ICA, subband power spectrum analysis, correlation analysis, and linear regression model. Detailed discussions of our experimental results are given in Section IV. Finally, we conclude our findings in Section V.

\section{SySTEM ARCHITECTURE}

A VR-based dynamic driving simulation environment is designed and built up for interactive driving experiments. It includes four major parts as shown in Fig. 1: 1) the 3-D highway driving scene based on the virtual reality technology; 2) the driving cabin simulator mounted on a 6-DOF dynamic Stewart motion platform; 3) the EEG measurement system with 36-channel EEG/EOG/ECG sensors; and 4) the proposed signal 


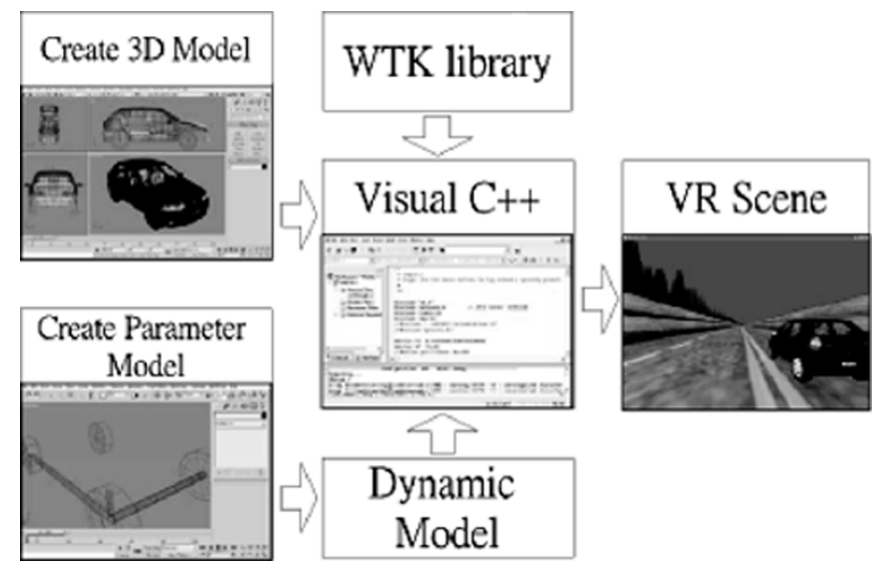

Fig. 2. Flowchart of the VR-based highway scene development environment. The dynamic models and shapes of the 3-D objects in the VR scene are created and linked to the WTK library to form a complete interactive VR simulated scene.

processing modules including ICA, power-spectral analysis, and linear regression model.

\section{A. VR-Based Driving Environment}

In this paper, a VR-based high-fidelity 3-D interactive highway scene and its emulation software, WorldToolKit (WTK) library and application programmer's interface (API) are developed [53]. The detailed development diagram of the VR-based scene is shown in Fig. 2. First, we create models of various objects (such as cars, roads, trees, etc.) for the scene and setup the corresponding positions, attitudes, and other parameters. Then, we develop the dynamic models among these virtual objects and build a complete simulated highway scene of full functionality using the high-level C-based API program.

The VR-based four-lane highway scene is projected on a $120^{\circ}$-surround screen $(304.1-\mathrm{cm}$ wide and $228.1-\mathrm{cm}$ high), which is $350 \mathrm{~cm}$ away from the driving cabin. The four lanes from left to right are separated by a median stripe. The distance from the left side to the right side of the road is equally divided into 256 points (digitized into values $0-255$ ), where the width of each lane and the car is 60 and 32 units, respectively. The refresh rate of highway scene was set properly to emulate a car driving at a fixed speed of $100 \mathrm{~km} / \mathrm{hr}$ on the highway. The car is randomly drifted (triggered from the WTK program and the on-set time is recorded) away from the center of the cruising lane to mimic the consequences of a nonideal road surface. The subject's driving error is defined as the deviation between the center of the vehicle and the center of the cruising (third) lane and it was continuously and simultaneously measured by the WTK program and recorded in the physiological measurement system accompanying with EEG/EOG/ECG physiological signals.

\section{B. Data Acquisition}

Thirty-three EEG/EOG channels (using sintered $\mathrm{Ag} / \mathrm{AgCl}$ electrodes with an unipolar reference at right earlobe), 2 ECG channels (bipolar connection), and the deviation between the center of the vehicle and the center of the cruising lane are simultaneously recorded by the Scan NuAmps Express system
(Compumedics Ltd., VIC, Australia). All the EEG/EOG sensors were placed based on a modified International 10-20 system. Before data acquisition, the contact impedance between EEG electrodes and scalp was calibrated to be less than $5 \mathrm{k} \Omega$. The EEG data were recorded with 16-bit quantization level at a sampling rate (SR) of $500 \mathrm{~Hz}$ and the recording are down-sampled to $\mathrm{SR}=250 \mathrm{~Hz}$ for the simplicity of data processing. Then EEG data were preprocessed using a simple low-pass filter with a cut-off frequency of $50 \mathrm{~Hz}$ to remove the line noise $(60 \mathrm{~Hz}$ and its harmonic) and other high-frequency noise for further analysis.

\section{Subjects}

It has been shown that human fatigue or drowsiness most commonly occurs late at night and during the afternoon. During these periods, alertness deficits would most likely take place in 1-h monotonous working [7], [8]. In this paper, we thus conducted all driving experiments in the early afternoon hours after lunch to maximize the opportunities to collect data during which subject driving performance became intermittent. All the subjects were instructed to keep the car at the center of the cruising lane by controlling the steering wheel. For each session, the subject started with a $15 \sim 45$-min calibration procedure and then was asked to drive the car continuously for $45 \mathrm{~min}$. The EEG/EOG/ECG data and the driving errors were measured and recorded simultaneously. Participants then returned on different days to complete the second 45-min driving session or the third session if necessary. We had collected successfully EEG data of 16 subjects (ages from 20 to $35 \mathrm{yr}$ ) participated in the VR-based driving task. We select participants who had two or more microsleep episodes based on the measured driving errors and confirmed by video recordings in at least two driving sessions for further analysis. Based on these criteria, five subjects (ten sessions) were selected for further modeling and cross-session testing.

\section{Drowsiness Measurement}

To find the relationship between the measured EEG signals and subject's cognitive state, and to quantify the level of the subject's alertness, we defined the subject's driving error index as the deviation between the center of the vehicle and the center of the cruising lane. Our pilot experimental studies showed that when the subject is drowsy (checked from video recordings), the driving error increases, and vice versa. Since the fluctuates of drowsiness level with cycle lengths were longer than 4 min [31], [32], [34], the driving errors were smoothed using a causal 90-s square moving-average filter advancing at 2-s steps to eliminate variance at cycle lengths shorter than 1-2 min.

\section{DATA ANALYSIS}

Fig. 3 shows the flowchart of the proposed signal processing procedure. The efficacy of using ICA to remove eye blinking and other artifacts such as muscle activity, line noise, and cardiac signals have been demonstrated in many studies [45]-[52]. Therefore, after collecting 33-channel EEG signals and driving deviations in a 45-min simulated driving session, the ICA algorithm is first used to remove a wide variety of artifacts. 


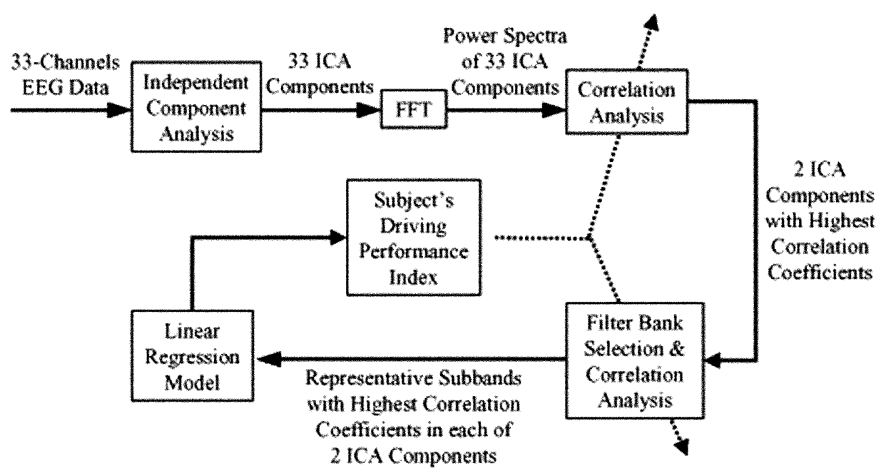

Fig. 3. Flowchart of data processing procedure for the drowsy estimation system.

Second, we calculate the normalized moving-averaged subband log power spectra of all 33 ICA components. The correlation coefficients between the smoothed subjects' driving error and the subband $\log$ power spectra of all ICA components at each frequency band are further evaluated to form a correlation spectrum. The normalized log subband power spectra of 2 ICA components with the highest correlation coefficients in some critical bands are further selected as the input features of the linear regression model to estimate the individual subject's driving performance. Detailed analyses are described in the following subsections.

\section{A. ICA}

Given an $N$-dimension data vector $X(t)=$ $\left[x_{1}(t) \cdots x_{N}(t)\right]^{T}$ observed at each time point $t$, the goal of ICA is to find a linear mapping matrix or unmixing matrix $W$ such that the unmixed components $Y(t)=\left[y_{1}(t) \cdots y_{N}(t)\right]^{T}$ of the linear transform $Y(t)=W X(t)$ of are statistically independent. The ICA methods were extensively applied to blind source separation problem since 1990s [36]-[44]. Subsequent technical reports [46]-[52] demonstrated that ICA was a suitable solution to the problem of EEG source segregation, identification, and localization based on the following assumptions.

1) The summation of different EEG source signals at the sensors is linear and instantaneous.

2) The signal source of muscle activity, eye, and cardiac signals are not time locked to the sources of EEG activity which is regarded as reflecting synaptic activity of cortical neurons [45]-[48].

By applying ICA algorithm to the EEG recorded from the scalp surface, we attempt to achieve the twin goals: removing EEG artifacts and extract EEG source signals associated with human fatigue or drowsiness index by the subject behaviors based on the assumptions that these signals are statistically independent.

In this paper, the observed data vector $X$ was first processed by "centering" to subtract its mean vector $\bar{X}$ as to make $X$ a zero-mean variable to simplify the ICA algorithm. After centering, the observed data vector $X$ is "whitened" to a new vector $\widetilde{X}$ to make its components uncorrelated and their variances equal unity, i.e., $E\left\{\tilde{X} \tilde{X}^{T}\right\}=I$. This can be done by using eigenvalue decomposition (EVD), $\widetilde{X}=V D^{-1 / 2} V^{T} X$, where $V$ is the orthogonal matrix of eigenvectors of $E\left\{\widetilde{X} \widetilde{X}^{T}\right\}$, and $D$ is the diagonal matrix of its eigenvalues. Whitening transforms the matrix $W$ into a new orthogonal matrix, $\widetilde{W}$. This can be seen from

$$
E\left\{\tilde{X} \widetilde{X}^{T}\right\}=I=E\left\{\left(\widetilde{W}^{-1} Y\right)\left(Y^{T} \widetilde{W}^{-T}\right)\right\}=E\left\{\widetilde{W}^{-1} \widetilde{W}^{-T}\right\}
$$

where $Y$ is the independent component. Then, the ICA algorithm was carried out with the "infomax" principle [40], [44], which is derived from a neural network $Y=g\left(W X+w_{0}\right)$ by maximizing the mutual information $I(Y, X)$ of the nonlinear output $Y$.

$$
I(Y, X)=H(Y)-H(Y \mid X)
$$

where $H(Y)$ is the entropy of the output, while $H(Y \mid X)$ is whatever entropy the output has which didn't come from the input $X$. In contrast with decorrelation techniques such as Principal Components Analysis (PCA), which ensures that $\left\langle y_{i} y_{j}\right\rangle=0, \forall i j$, ICA imposes the much stronger criterion by finding the multivariate probability density function of $Y$ factorized: $f_{y}(y)=\prod_{i=1}^{N} f_{y_{i}}\left(y_{i}\right)$. When $g(x)$ is monotonically increasing or decreasing, (i.e., has a unique inverse), the p.d.f. of the output, $f_{y}(y)$ can be written as a function of the p.d.f. of the input $f_{x}(x)$ as

$$
f_{y}(y)=\frac{f_{x}(x)}{\left|\frac{\partial y}{\partial x}\right|} .
$$

The entropy of the output $H(y)$ is given by

$$
H(y)=E\left[\ln \left|\frac{\partial y}{\partial x}\right|\right]-E\left[\ln f_{x}(x)\right]
$$

In order to maximize to the entropy of $y$, which makes $\left\{y_{i}\right\}$ more independent, by changing $w$, we need to maximize the first term of (3), where the second term on the right may be considered to be unaffected by alterations in a parameter $w$ determining $g(x)$. This can be done by considering the "training set" of " $x$ " to approximate the density, $f_{x}(x)$, and deriving an "online" stochastic gradient ascent rules for adjusting $W$ based on the infomax principle [40].

For one input and one output with logistic transfer function $g(u)=\left(1+e^{-u}\right)^{-1}$

$$
\Delta w \propto \frac{\partial H}{\partial w}=\frac{\partial}{\partial w}\left(\ln \left|\frac{\partial y}{\partial x}\right|\right)=\left(\frac{\partial y}{\partial x}\right)^{-1} \frac{\partial}{\partial w}\left(\frac{\partial y}{\partial x}\right)
$$

where $\partial y / \partial x=w y(1-y)$ and $(\partial / \partial w)(\partial y / \partial x)=$ $y(1-y)(1+w x(1-2 y))$. Then $\Delta w$ is calculated as

$$
\Delta w \propto \frac{1}{w}+x(1-2 y) .
$$

For $N$-input and $N$-output network, the learning rules are similar in form

$$
\Delta W \propto\left[W^{T}\right]^{-1}+(1-2 Y) X^{T}
$$




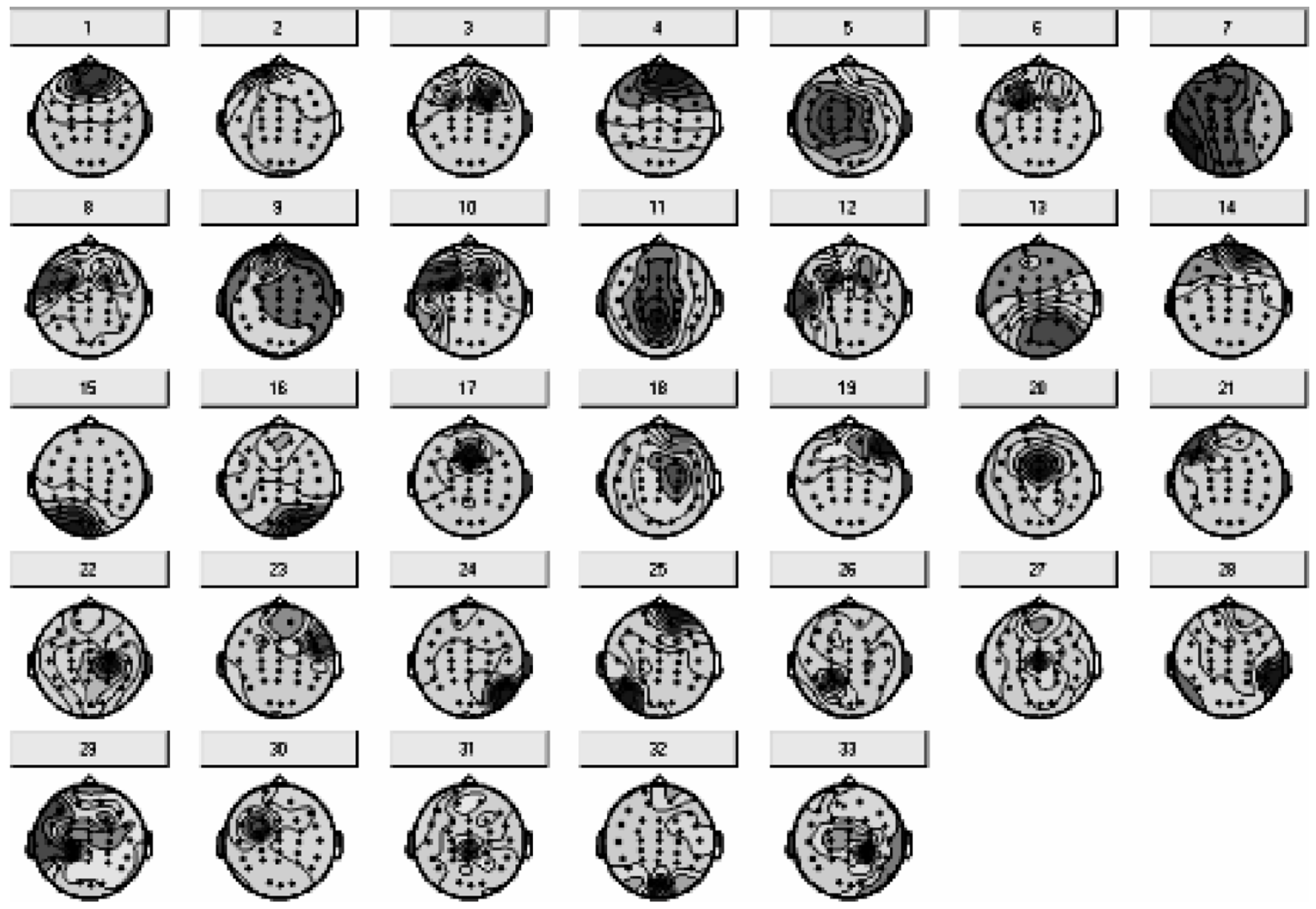

Fig. 4. Scalp topography of ICA weighting matrix $W^{-1}$ of 33 ICA components.

or for individual weight $w_{i j}$ of $W$

$$
\Delta w_{i j} \propto \frac{\operatorname{cof}\left(w_{i j}\right)}{\operatorname{det} W}+x_{j}\left(1-2 y_{i}\right)
$$

where cof $\left(w_{i j}\right)$ is the cofactor of $w_{i j}$ which is $(-1)^{i+j}$ times the determinant of the matrix obtained by removing the $i^{\text {th }}$ row and $j^{t h}$ column from $W$. More details can be referenced to [40].

In this paper, the ICA weighting matrix $W$ can be obtained using learning rule (6) and (7). After ICA training on 33-channel EEG data in our case, we obtain 33 ICA components. The back projection of the ICA components to the EEG signals can be done by $X=W^{-1} Y$

$$
\begin{aligned}
X(t) & =\left[\begin{array}{c}
x_{1}(t) \\
x_{2}(t) \\
\vdots \\
x_{33}(t)
\end{array}\right] \\
& =W^{-1} Y(t) \\
& =\left[\begin{array}{c}
w_{1,1} \\
w_{2,1} \\
\vdots \\
w_{33,1}
\end{array}\right] y_{1}(t)+\left[\begin{array}{c}
w_{1,2} \\
w_{2,2} \\
\vdots \\
w_{33,2}
\end{array}\right] y_{2}(t)+\cdots+\left[\begin{array}{c}
w_{1,33} \\
w_{2,33} \\
\vdots \\
w_{33,33}
\end{array}\right] y_{33}(t)
\end{aligned}
$$

where the columns of the inverse matrix $W^{-1}$ give the projection strengths of the respective components onto the scalp sensors. The scalp topographies of the components provide evidence for their biological origin (e.g., eye activity should project mainly to frontal sites, and the drowsiness-related potential is on the parietal lobe to occipital lobe, etc.). In general, corrected EEG signals can then be derived as $\hat{X}=W^{-1} \hat{Y}$, where $\hat{Y}$ is the matrix of activation waveforms $Y$, with rows representing artifactual sources set to zero. Fig. 4 shows the scalp topographies of ICA back-projection matrix $W^{-1}$ of Subject 3. As shown in Fig. 4, most of the EEG artifacts and channel noises in EEG recordings are effectively separated into ICA components 1 and 4, while ICA components 5, 11, and 13 (selected by visual inspection) may be considered as effective "sources" associated with drowsiness in the VR-based driving experiment. Further check was performed in the Section IV.

\section{B. Power-Spectrum Analysis}

Analysis of changes in spectral power and phase can characterize the perturbations in the oscillatory dynamics of ongoing EEG. Applying such measures to the activity time courses of separated independent component sources avoids confounds caused by miscancellation of positive and negative potentials from different sources to the recording electrodes, and by misallocation to the recording electrodes activity that originates in various and commonly distant cortical sources. Fig. 5 shows the diagram of moving-average power spectral analysis [54] for a 


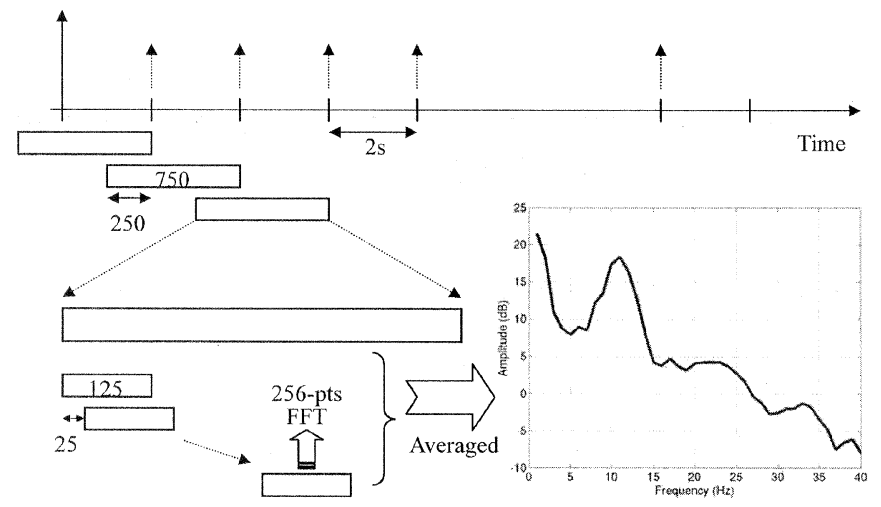

Fig. 5. Moving-averaged log power-spectral analysis for ICA/EEG signals.

single ICA component, which was obtained from decomposition of 33 channels of the EEG signals. The time series of the single ICA component was first divided into 750-point epochs using Hanning window in (9) with 250-point overlap, i.e., stepping in $2 \mathrm{~s}$. Each 750-point epoch was further divided into several 125-point frames using Hanning windows with 25-point step size again.

$$
w(n)=0.54-0.46 \cos \left(\frac{2 \pi n}{L-1}\right), \quad 0 \leq n \leq L-1
$$

Each 125-point frame was extended to 256 points by zero-padding to calculate its power spectrum by using a 256-point fast Fourier transform (FFT), resulting in power-spectrum density estimation with a frequency resolution near $1 \mathrm{~Hz}$. Then we averaged the power spectrum of all the subepochs within each epoch. Previous studies [55], [56] show that the transient amplitudes of EEG power spectrum involved in wake-sleep regulation are very different. The cortex produces low amplitude and fast oscillations during waking, and generates high-amplitude, slow cortical oscillations during the onset of sleep. Their reports also showed that the EEG spectral amplitudes correlated with the wake-sleep transition more linearly in the logarithmic scale than in the linear scale. Our previous study [62] based on the same task and empirical results in this study also confirm this phenomenon. Therefore, the averaged power spectrum of each epoch was normalized to logarithmic scale to linearize these multiplicative effects. The resultant power-spectrum time series of single ICA component for each 45-min session consisted 40 frequency bins (from 0.98 to $39.1 \mathrm{~Hz}$ ) stepping at 2-s time intervals. Finally, a median filtering using a moving averaged 90-s window was applied to the power spectrum to further minimize the presence of artifacts in the ICA/EEG signals and to match the time intervals of the driving performance index. The same procedure of power-spectrum analysis was applied to all 33 ICA components and other 33 EEG channels for comparisons.

\section{Correlation Coefficient}

In order to find the relationship between the brain activities and subject's driving performance, and to quantify the level of
Power Changes in $10 \mathrm{~Hz}$ in ICA Components $11(\mathrm{~L})$ and $13(\mathrm{R})$

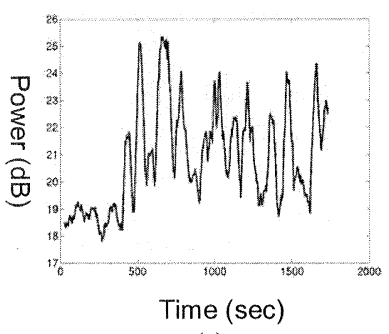

(a)

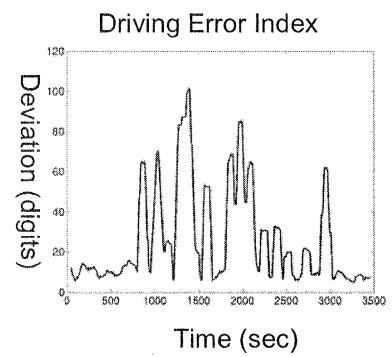

(c)

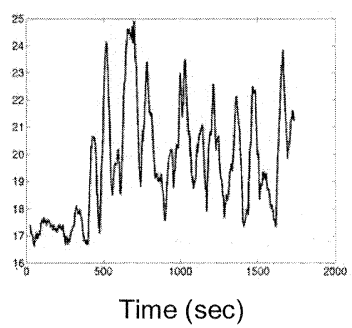

(b)

Correlation Spectrum of ICA components 11 and 13

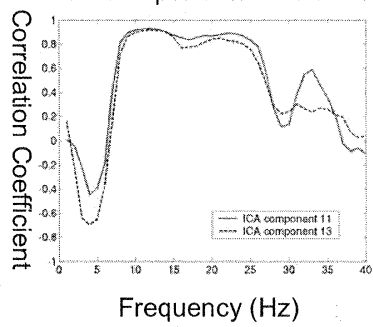

(d)
Fig. 6. Fluctuations in the driving error index and concurrent changes in power spectrum of ICA components and their corresponding correlation spectrum. (a) and (b) Changes of power spectrum in $10 \mathrm{~Hz}$ with time of the ICA components 11 and 13 of Subject 3 after 90-s moving-average spectral analysis. (c) The smoothed 90-s driving error index. (d) Correlation coefficients from $1-40 \mathrm{~Hz}$ forming a correlation spectrum of the ICA components 11 and 13 .

the subject's drowsiness, we computed the correlation coefficient between the time course of the fluctuations in driving error and the concurrent changes in the ICA spectrum of EEG signals by using the Pearson Correlation Coefficient

$$
C C_{i}(k)=\frac{\sum_{n}(d p(n)-\overline{d p})\left(p_{i}(n, k)-\overline{p_{i}(k)}\right)}{\sqrt{\sum_{n}(d p(n)-\overline{d p})^{2} * \sum_{n}\left(p_{i}(n, k)-\overline{p_{i}(k)}\right)^{2}}}
$$

where $d p(n)$ is the time series of the driving performance index, $p_{i}(n, k)$ is the time-frequency series of the $i$ th ICA component, $n$ is the time stepping size in $2 \mathrm{~s}$, and $k$ is the frequency index $k=1,2, \ldots, 40$. The $C C_{i}(k)$ forms a correlation spectrum that measures correlation between changes in the ICA/EEG log power spectrum, $p_{i}(n, k)$, of the $i$ th ICA component for the $k$ th frequency index and the subject performance index $d p(n)$. The variables, $\overline{d p}$ and $\overline{p_{i}(k)}$ (related to the $k$ th frequency index) are the expected values of $d p(n)$ and $p_{i}(n, k)$, respectively. Fig. 6(a) and (b) show an example of the log spectral changes at $10 \mathrm{~Hz}$ of components 11 and 13 of Subject 3. Fig. 6(c) shows the minutescale fluctuation of the driving performance index in a 45-min driving session. Note that the fluctuations in the driving error index change slowly in minute scales, which was consistent with previous studies [31], [34] in an auditory detection task. We then calculate the correlation coefficient between the time series of the driving performance index and the concurrent changes in the power spectrum in components 11 and 13 using (9) for each frequency. Results for 40 frequencies between 0.98 and $39.1 \mathrm{~Hz}$ are shown Fig. 6(d). 


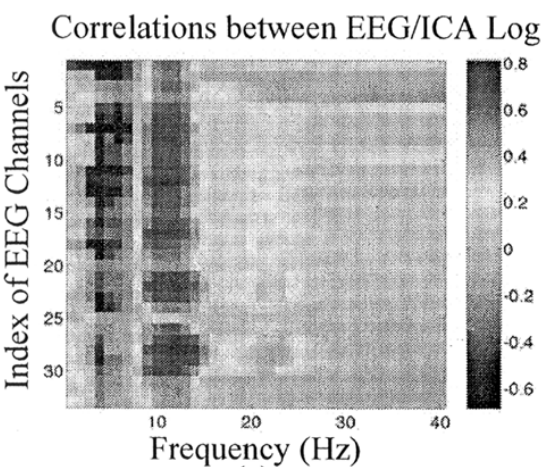

(a)

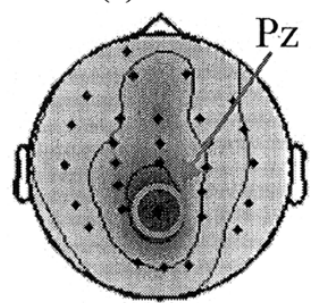

(c)

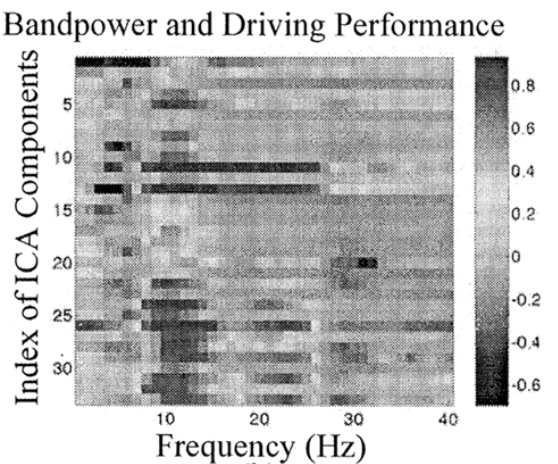

(b)

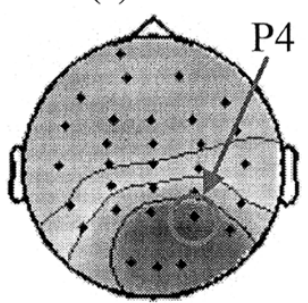

(d)

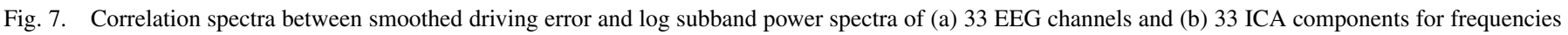

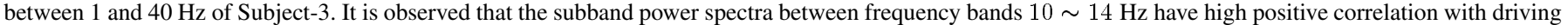

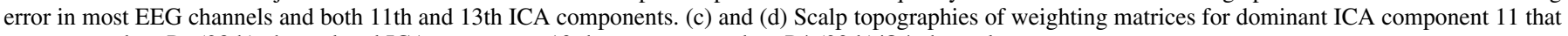
was centered on $\mathrm{Pz}$ (28th) channel and ICA component 13 that was centered on P4 (29th)/O4 channels.

\section{RESULTS AND DISCUSSIONS}

\section{A. Relationship Between the ICA/EEG Power Spectrum and Drowsiness}

After ICA training and analysis of log subband power spectrum for each ICA components/EEG channels, we computed the spectral correlations between changes in the ICA/EEG log subband power spectrum and driving performance by computing the correlation coefficients between the two time series at each frequency band. Fig. 7 shows the resulting correlation spectra of Subject 3 in (a) 33 EEG channels and (b) 33 ICA components. The horizon axis indexes frequency bands between 1 and $40 \mathrm{~Hz}$ and the vertical axis indexes the EEG channels/ICA components. In Fig. 7(a), the correlation spectrum shows a strong positive correlation between fluctuations in EEG bandpower of frequency bands between 10 and $14 \mathrm{~Hz}$ across most of the EEG channels. As driving error increases, so does EEG bandpower. Strong correlations also appear between ICA log power spectrum and subject driving performance [Fig. 7(b)]. For example, the log power spectra of components 11 and 13 between 9-25 Hz are strongly positively correlated with subject driving performance. Fig. 7(c) and (d) shows the scalp topographies of components 11 (Pz-dominant) and 13 (P4 dominant). The correlations are particularly strong at central and posterior areas, which are consistent with previous studies in the driving experiments [19], [21], [29]. The relatively high correlation coefficients near $\alpha$-band $(8-13 \mathrm{~Hz})$ suggests that alpha band frequencies $(8-13 \mathrm{~Hz})$ may be suitable for drowsiness (microsleep) estimation, as the subject's cognitive state might fall into stage one of the NREM sleep. Next, we compared correlation spectra from different subjects to see if

\begin{tabular}{|c|c|c|c|c|c|}
\hline $\begin{array}{c}\text { ICA } \\
\text { component }\end{array}$ & 11 & 13 & 26 & 24 & 5 \\
\hline $\begin{array}{c}\text { Scalp } \\
\text { topography }\end{array}$ & & & & \\
\hline $\begin{array}{c}\text { Correlation } \\
\text { coefficient }\end{array}$ & 0.92 & 0.91 & 0.88 & 0.82 & 0.80 \\
\hline
\end{tabular}

\begin{tabular}{|c|c|c|c|c|c|}
\hline \multicolumn{6}{|c|}{ (a) Subject-3 } \\
\hline $\begin{array}{c}\text { ICA } \\
\text { component }\end{array}$ & 17 & 8 & 33 & 19 & 11 \\
\hline $\begin{array}{c}\text { Scalp } \\
\text { topography }\end{array}$ & & & & & \\
\hline $\begin{array}{l}\text { Correlation } \\
\text { coefficient }\end{array}$ & 0.92 & 0.91 & 0.89 & 0.84 & 0.72 \\
\hline
\end{tabular}

(b) Subject-2

Fig. 8. Correlation spectra between smoothed driving errors and log subband power spectra of first 5 ICA components of (a) Subject 3 and (b) Subject 2, respectively. It is observed that the first 2 ICA components of Subjects 3 and 2 are somewhat different in their scalp topography but within the ambit of parietal lobe and occipital lobe.

the relationship between IAC power and driving performance is stable across subjects. Fig. 8 shows the scalp topographies and spectrum correlation between smoothed driving performance and $\log$ subband power spectra of top five ICA components of Subject 3 and Subject 2. As can been seen, that the best drowsiness-correlated components (best matching) differ in Subjects 2 and 3, in general their scalp topographies are all within the ambit of central lobe to occipital lobe. This result is consistent with the subject variability in the relationship between scalp EEG power and subject task performance reported in [31] and [62]. 
TABLE I

CORRElation CoEFFicients BetweEn Log SubBand POWER SPECTRA AND DRIVING ERROR OF SUBJECT 3 CORRESPONDING TO DIFFERENT FREQUENCY BANDS FROM 8 TO 15 Hz OF ICA COMPONENT 11 AND 13 IN Training AND Testing SEssions Using SAmE ICA WeIGHTING MATRICES ObTAINED FROM TRAINING SESSION

\begin{tabular}{|c|c|c|c|c|c|c|c|c|c|}
\hline \multicolumn{2}{|c|}{$\begin{array}{l}\text { Band } \\
\text { ICA }\end{array}$} & $8 \mathrm{~Hz}$ & $9 \mathrm{~Hz}$ & $10 \mathrm{~Hz}$ & $11 \mathrm{~Hz}$ & $12 \mathrm{~Hz}$ & $13 \mathrm{~Hz}$ & $14 \mathrm{~Hz}$ & $15 \mathrm{~Hz}$ \\
\hline \multirow{4}{*}{ Component Index } & & & & & & & & \\
\hline \multirow{4}{*}{ Com 11 } & Training & 0.82 & 0.89 & 0.92 & 0.92 & 0.92 & 0.92 & 0.89 & 0.87 \\
\cline { 2 - 11 } & Testing-1 & 0.86 & 0.88 & 0.88 & 0.88 & 0.87 & 0.86 & 0.83 & 0.82 \\
\cline { 2 - 11 } & Testing-2 & 0.79 & 0.87 & 0.90 & 0.92 & 0.91 & 0.91 & 0.86 & 0.78 \\
\cline { 2 - 11 } & Testing-3 & 0.78 & 0.90 & 0.93 & 0.93 & 0.93 & 0.94 & 0.94 & 0.91 \\
& Training & 0.77 & 0.88 & 0.90 & 0.91 & 0.92 & 0.91 & 0.90 & 0.86 \\
\cline { 2 - 11 } & Testing-1 & 0.87 & 0.90 & 0.90 & 0.89 & 0.88 & 0.87 & 0.84 & 0.80 \\
\cline { 2 - 11 } & Testing-2 & 0.75 & 0.87 & 0.87 & 0.90 & 0.90 & 0.88 & 0.85 & 0.79 \\
\cline { 2 - 11 } & Testing-3 & 0.76 & 0.89 & 0.91 & 0.92 & 0.93 & 0.92 & 0.92 & 0.89 \\
\hline
\end{tabular}

TABLE II

Correlation CoefFicients Between log Subband Power Spectra AND Driving ERror of SubJect 3 Using FIVE BESt Frequency BANDS (From 10 TO 14 Hz) CORRESPONDING TO DIFFERENT Single ICA COMPONENT. SAme ICA Weighting Matrices ObTained From Training SESSION WERE USED FOR TESTING SESSION PERFORMED ON OTHER DAY

\begin{tabular}{|c|c|c|c|c|c|c|c|}
\hline ICA component & 5 & 11 & 13 & 24 & 26 & 29 & 31 \\
\hline Training & 0.80 & 0.92 & 0.91 & 0.82 & 0.88 & 0.78 & 0.78 \\
\hline Testing & 0.84 & 0.93 & 0.92 & 0.82 & 0.89 & 0.82 & 0.79 \\
\hline
\end{tabular}

\section{B. Selection of Frequency Bands Based on Spectral Analysis and Driving Errors}

Our previous studies [62], based on the same driving task, showed that it is not optimal to use full EEG frequency bands to accurately estimate individual changes in vigilance and driving error because the pervasive artifacts could contaminate some frequency bandpowers in EEG dynamics. In this paper, therefore, we decided to choose the best EEG channel(s) and frequency band(s) that most correlated with drowsiness level of subjects to estimate the fluctuation in subject driving performance.

In this section, we compared the correlation between log subband power spectra and driving error for each frequency bands and individual ICA component to find the optimal subbands and localizations of electrodes according to the scalp topographies of ICA weighting matrices. Table I shows the correlation coefficients between the power spectrum of ICA components 11 and 13 at different frequency bands and the driving performance of Subject 3 across different driving sessions. The ICA unmixing matrix was obtained by training ICA with the training set and tested against testing sessions collected in different days from the same subject. For Subject 3, time courses of ICA power spectra of components 11 and 13 best correlated with subject driving performance. The strongest correlation between ICA power and driving performance occur between 10 and $14 \mathrm{~Hz}(r=0.94)$ as shown in Table I. Table II lists the spectrum correlation at $10-14 \mathrm{~Hz}$ of components $5,11,13,24$, 26,29 , and 31 in both training and testing sessions. Both components exhibit strong alpha activity which was associated with microsleep at occipital and central sites. Table III lists the two
TABLE III

OPTIMAL 2 ICA COMPONENTS AND FREQUENCY-BAND RANGES CORRESPONDING TO DIFFERENT SUBJECTS ACCORDING TO THE Higher Correlation CoEfFicients Between Log SubBand POWER SPECTRA AND DRIVING PERFORMANCE

\begin{tabular}{|c|c|c|c|c|c|}
\hline Subject & Subject 1 & Subject 2 & Subject 3 & Subject 4 & Subject 5 \\
\hline ICA Components & 17,28 & 17,8 & 11,13 & 4,5 & 22,25 \\
\hline Bands & $5-9 \mathrm{~Hz}$ & $8-12 \mathrm{~Hz}$ & $10-14 \mathrm{~Hz}$ & $4-8 \mathrm{~Hz}$ & $8-12 \mathrm{~Hz}$ \\
\hline
\end{tabular}

best drowsiness-related ICA components and their best-correlated frequency bands in different subjects. The relationship between minute-scale changes in driving performance and power spectrum appears variable across subjects.

The above analyses provide strong and converging evidence that changes in subject alertness level indexed by driving performance in a driving task are strongly correlated with the changes in the ICA power spectrum at several frequencies at predominantly central and posterior cites. This relationship is stable over time in different sessions from the same subject, but relatively variable between subjects. These results are consistent with the findings from a simple auditory target detection task reported in [31], [32]. These findings suggest that for maximal accuracy the estimation algorithm should be capable of adapting to individual differences in the mapping between EEG and alertness.

\section{Drowsiness Estimation Based on EEG Log Bandpower}

An early study [31] of alertness monitoring based on a simple auditory detection task demonstrated the feasibility of using linear regression and neural network to estimate the time course of detection error rate from changes in EEG power. A nature question is to what extent an index of alertness based on auditory detection performance can estimate fluctuations in performance on more realistic and complicated tasks such as driving. In this section, we investigate the feasibility of accurately estimating subject driving performance in a VR-based driving simulation experiments. Results of this study will also serve as the baseline/standard for justifying the development of more sophisticated alertness-monitoring method based on linear regression model applied to power spectral data of ICA components (as opposed to EEG channels).

The linear regression model attempts to model the relationship between the selected input features $X$ to the observed output data $y_{d}$ at each sampled data point. i.e., given the input data set $X$ and desired output data $y_{d}$ at each data point, the least-squared linear regression method is to find an optimal parameter set $\{A, b\}$, such that $y=A X+b$, subjected to minimizing the squared error cost function $\varepsilon=\Sigma\left(y_{d}-y\right)^{2}$.

We first need to decide the number of EEG channels and frequency bins to use in the multivariate a linear regression model. For practical and routine application, EEG-based cognitive assessment systems should use as fewer EEG sensors as possible to reduce the preparation time for device wiring and computational cost for continuous alertness level estimation in near real time. Previous studies [31], [34] demonstrated it is feasible to accurately estimate subject performance based on as few as two EEG channels which is statistically significantly better than using only one EEG site. Therefore, in this paper we use two out of 33 EEG sites. However, due to individual variability, we 
TABLE IV

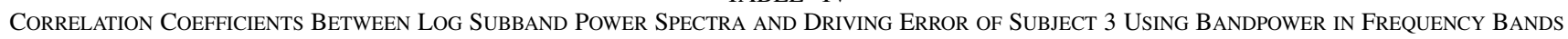
From 10 TO 14 Hz Corresponding to DifFERENT Single EEG Channel in Training/TeSting SESSION

\begin{tabular}{|c|c|c|c|c|c|c|c|}
\hline Index of EEG Channels & $\mathrm{Fz}$ & $\mathrm{FCz}$ & $\mathrm{Cz}$ & $\mathrm{CPz}$ & $\mathrm{P} 3$ & $\mathrm{Pz}$ & $\mathrm{P} 4$ \\
\hline Training & 0.67 & 0.69 & 0.69 & 0.72 & 0.69 & 0.77 & 0.72 \\
\hline Testing & 0.75 & 0.77 & 0.77 & 0.78 & 0.74 & 0.80 & 0.79 \\
\hline
\end{tabular}

TABLE V

Optimal 2 EEG Channels AND the AsSOCIATED FREQUENCY-BAND RANGES CORRESPONDING TO DIFFERENT SUBJECTS BASED ON CENTRAL ELECTRODE

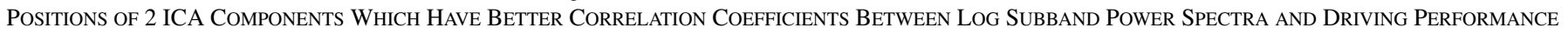

\begin{tabular}{|c|c|c|c|c|c|}
\hline & Subject 1 & Subject 2 & Subject 3 & Subject 4 & Subject 5 \\
\hline Bands & $5-9 \mathrm{~Hz}$ & $8-12 \mathrm{~Hz}$ & $10-14 \mathrm{~Hz}$ & $4-8 \mathrm{~Hz}$ & $8-12 \mathrm{~Hz}$ \\
\hline EEG Channels & $\mathrm{O} 1, \mathrm{O} 2$ & $\mathrm{CPz}, \mathrm{Pz}$ & $\mathrm{Pz}, \mathrm{P} 4$ & $\mathrm{O} 1, \mathrm{O} 2$ & $\mathrm{P} 3, \mathrm{O} 1$ \\
\hline
\end{tabular}

TABLE VI

Driving ERror Estimation USING TOTAL TEN Frequency BANDS (5 FOR EACH EEG CHANNEL) AS INPUT FEATURES OF THE LINEAR REGRESSION MODEL FOR FIVE SUBJECTS

\begin{tabular}{|c|c|c|c|c|c|c|}
\hline & Subject 1 & Subject 2 & Subject 3 & Subject 4 & Subject 5 & Average \\
\hline Training & $68 \%$ & $90 \%$ & $91 \%$ & $80 \%$ & $94 \%$ & $84.6 \%$ \\
\hline Testing & $78 \%$ & $86 \%$ & $87 \%$ & $88 \%$ & $73 \%$ & $82.4 \%$ \\
\hline
\end{tabular}

Driving Performance Estimation of Subject-3 Using Linear Regression Model

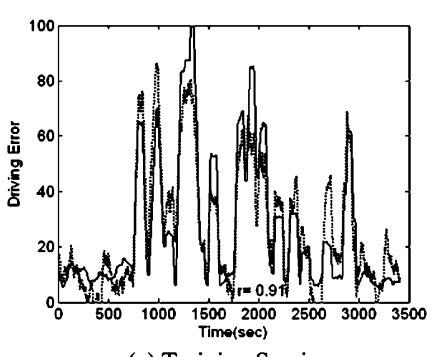

(a) Training Session

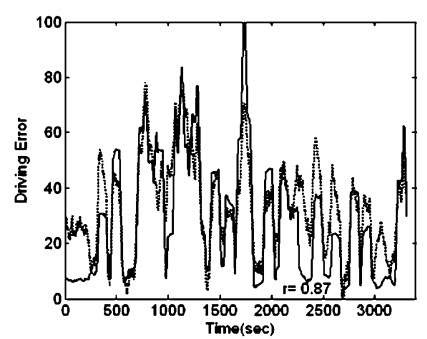

(a) Testing Session
Fig. 9. Driving error estimates for training/testing sessions of Subject 3, based on a linear regression model (solid line) with subband log power spectra at frequency bands $10 \sim 14 \mathrm{~Hz}$ of EEG channels $\mathrm{Pz}$ and $\mathrm{P} 4$ (selected according to Table V), overplotted against actual driving error time series for the session (dotted line). The correlation coefficient between the two time series is $r=0.91$ in the training session and $r=0.87$ in the testing session.

need to, for each subject, select five frequency bins of the best two EEG channels as inputs to multivariate linear regression models. As an example, Table IV shows, for Subject 3, the correlation coefficients between the log subband power spectra and the driving error based on frequency bands from 10 to $14 \mathrm{~Hz}$ at $\mathrm{Fz}, \mathrm{FCz}, \mathrm{Cz}, \mathrm{CPz}, \mathrm{P} 3, \mathrm{Pz}$, and P4 in the training/testing session. Comparing to the results of using ICA components, the correlation coefficients are in general lower than the values in Table II, partially due to pervasive artifacts and other noises. In this example, $\mathrm{Pz}$ and $\mathrm{P} 4$ channels were selected for further linear regression analysis. Fig. 9 plots the resultant driving performance estimate based on a linear regression model applied to the power-spectral time series at $10-14 \mathrm{~Hz}$ of $\mathrm{Pz}$ and $\mathrm{P} 4$. The correlation coefficient between estimated and actual driving performance is 0.91 in the within-session testing and 0.87 in the cross-session testing. Table $\mathrm{V}$ lists the optimal two EEG channels and associated frequency bands for different subjects. Table VI shows the accuracy of driving performance estimation for all five subjects. The mean correlation coefficient be- tween actual driving error time series in within- and cross-session testing is 0.846 and 0.824 , respectively.

\section{Drowsiness Estimation Based on Log Bandpower of ICA Components}

In this paper, we use a least-square multivariate linear regression model [60], [61] to estimate the subject's driving error based on the information obtained from the subband powerspectra analysis of ICA components. In our paper, we used only two ICA components that exhibit the highest correlation between the ICA subband power spectrum and the driving performance to remove the contamination from EEG artifacts and extract drowsiness-related brain activity. Results of our correlational analysis presented above suggest we can use the power spectrum as few as two components at five frequency bins (e.g., 10-14 Hz for ICA component 11 for Subject 3) as the input data $X$ of linear regression model. After training process, we get the optimal parameters $\{A, b\}$ such that the output of the linear regression model, $y=A X+b$, best matches the observed output (driving performance, $\left.y_{d}\right)$ with minimal $\varepsilon=\Sigma\left(y_{d}-y\right)^{2}$. For each subject who participated in two-three driving experiments, the ICA unmixing matrix obtained from the training sessions was used to spatially filter the features in the testing sessions so that all data were processed in the same way for the same subject before feeding to the estimation models. The linear regression model was trained on one session and tested on the other session for each subject. Fig. 10 plots the estimated and actual driving error of training/testing sessions of Subject 3. The linear regression model in this figure is trained with one session and tested against the training session (within-session) and a separated session (cross-session testing). As can been seen, the estimated driving error matched well with the actual driving performance $(r=0.93$ ) in the within-session testing and $r=0.92$ in the cross-session testing, which are higher than those using power spectrum of two EEG scalp channels shown in Fig. 9. 
TABLE VII

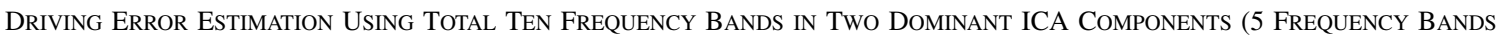
FOR EACH ICA COMPONENT) AS INPUT FEATURES OF LINEAR REGRESSION MODEL FOR FIVE SUBJECTS

\begin{tabular}{|c|c|c|c|c|c|c|}
\hline & Subject 1 & Subject 2 & Subject 3 & Subject 4 & Subject 5 & Average \\
\hline Training & $77 \%$ & $91 \%$ & $93 \%$ & $80 \%$ & $90 \%$ & $86.2 \%$ \\
\hline Testing & $91 \%$ & $89 \%$ & $92 \%$ & $89 \%$ & $80 \%$ & $88.2 \%$ \\
\hline
\end{tabular}

Driving Performance Estimation of Subject-3 Using Linear Regression Model

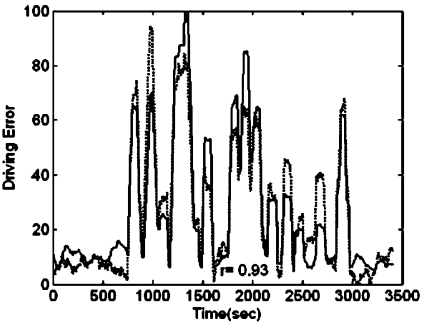

(a) Training Session

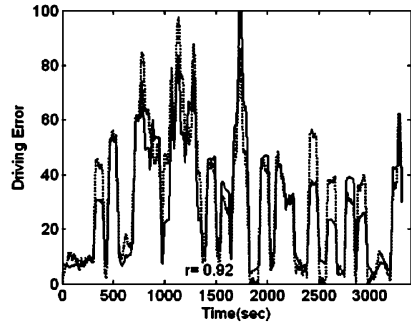

(a) Testing Session
Fig. 10. Driving error estimates for training/testing sessions of Subject 3, based on a linear regression model (solid line) with subband log power spectra at frequency bands $10 \sim 14 \mathrm{~Hz}$ of ICA components 11 and 13 selected according to Table III, overplotted against actual driving error time series for the session (dotted line). The correlation coefficient between the two time series is $r=0.93$ in the training session and $r=0.92$ in the testing session.

Driving Performance Estimation of Subject-2 Using Linear Regression Model

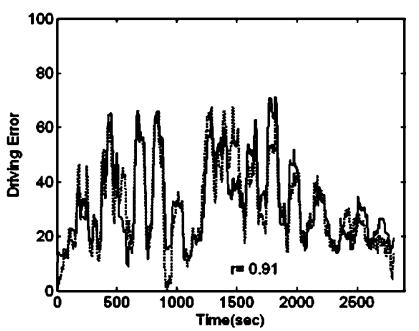

(a) Training Session

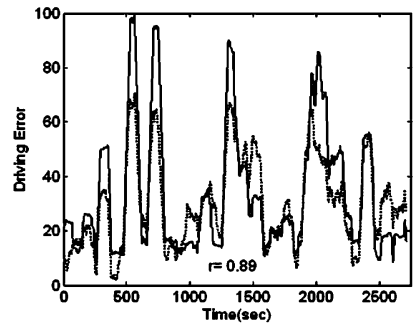

(a) Testing Session
Fig. 11. Driving error estimates for training/testing sessions of Subject 2 , based on a linear regression model (solid line) with subband log power spectra at frequency bands $8 \sim 12 \mathrm{~Hz}$ of ICA components 8 and 17 selected according to Table III, overplotted against actual driving error time series for the session (dotted line). The correlation coefficient between the two time series is $r=0.91$ in the training session and $r=0.89$ in the testing session.

Fig. 11 demonstrates the results of driving performance estimation for Subject 2. Again, the performance estimate matched well with the actual driving performance $(r=0.91)$ in the within-session testing and $r=0.89$ in the cross-session testing. Table IV shows the statistics across ten sessions for five subjects. The mean correlation coefficient between estimated and actual driving performance is $0.862 \pm 0.072$ for within-session testing and $0.882 \pm 0.048$ for cross-session testing. These results suggest that continuous ICA-based driving performance estimation using a small number of frequency bands is feasible, and can provide accurate information about minute-to-minute changes in operator's alertness.

Finally, we compared the accuracy of the proposed ICA-component based estimates to those produced by the best EEG-based standards. Comparing Tables VI and VII, the accuracies of estimates based on ICA spectrum are consistently better than those based on scalp-recorded EEG spectrum; expect the within-session testing in Subject 5. This result justifies the further development of the proposed method. However, for practice and routine application or in some applications where the number of available EEG channels is limited, EEG-based cognitive assessment systems should use as fewer EEG sensors as possible to reduce the preparation time for device wiring and computational cost for continuous alertness level estimation in near real time. According to the analysis shown in Tables III-VII, we believe it is adequate to use the only 2 EEG channels at central positions of the two effective ICA components to assess the alertness level of subjects continuously when the number of EEG sensors is not sufficient for ICA decomposition which usually requires more than ten channels of simultaneously recorded data.

\section{CONCLUSION}

In this paper, an EEG-based drowsiness estimation technology based on ICA, power-spectrum analysis, correlation analysis, and the linear regression model is proposed and evaluated in a VR-based driving environment. We demonstrated a close relationship between fluctuations in driving performance and the log subband power ICA/EEG spectrum. This relationship appears stable within individuals across sessions, but is somewhat variable between subjects. Our experimental results show that the proposed analysis methods are feasible to accurately estimate individual driving error accompanying loss of alertness by linear regression model applied to ten subband log power spectra near $\alpha$-bands of 2 ICA components as inputs. Averaged accuracies of within- and cross-session estimation for five subjects are $86.2 \%$ and $88.2 \%$, respectively. We also compared the results to those obtained by linear regression models applied to two best drowsiness-related EEG channels located at central electrodes of the corresponding ICA components. Average accuracies of within- and cross-session estimation for five subjects are $84.6 \%$ and $82.4 \%$, respectively. Although the accuracy is somewhat lower than those using ICA components, it does not require collecting more EEG channels data for ICA training. Thus, this approach might be advantageous in certain applications where the number of available EEG channels is limited. Both methods can lead to online portable embedded systems for noninvasive monitoring of the cognitive state of human operators in attention-critical settings.

\section{ACKNOWLEDGMENT}

The authors would like to thank J.-R. Duann, Y.-C. Chen, T.-Y. Huang, S.-C. Guo, H.-H. Lee, and H.-W. Tung for their great help with developing and operating the experiments. 


\section{REFERENCES}

[1] NHTSA. Drowsly driver detection and warning system for commercial vehicle drivers: Field proportional test design, analysis, and progress. National Highway Traffic Safety Administration, Washington, DC. [Online]. Available: http://www.nhtsa.dot.gov/

[2] NSF. Sleep facts and stats. National Sleep Foundation, Washington, DC. [Online]. Available: http://www.sleepfoundation.org/

[3] Drowsydriving. Drowsy driving surveys. Drowsydriving, Washington, DC. [Online]. Available: http://www.drowsydriving.org

[4] AAAFoundation. Drunk or drowsy?- Study finds may police officers mistake tired drivers for drunk drivers. AAA Foundation, Washington, DC. [Online]. Available: http://www.aaafoundation.org

[5] L. Y. Chang and F. Mannering, "Analysis of injury severity and vehicle occupancy in truck-nontruck-involved accidents," Accident Anal. Prevent., vol. 31, pp. 579-592, 1999.

[6] L. P. Kostyniuk, F. M. Streff, and J. Zakarajsek, "Identifying unsafe driver actions that lead to fatal car-truck crashes," AAA Found. Traffic Safety, 2002.

[7] J. Hendrix, "Fatal crash rates for tractor-trailers by time of day," in Proc. Int. Truck and Bus Safety Res. Policy Symp., 2002, pp. 237-250.

[8] H. Ueno, M. Kaneda, and M. Tsukino, "Development of drowsiness detection system," in Proc. 1994 Vehicle Navigation and Information Systems Conf., vol. 31, Sep. 1994, pp. 15-20.

[9] G. Hamouda and F. F. Saccomanno, "Neural network model for truck driver fatigue accident detection," in Proc. 1995 Electrical and Computer Engineering Canadian Conf., vol. 1, Sep. 1995, pp. 362-365.

[10] M. Eriksson and N. P. Papanikotopoulos, "Eye-tracking for detection of driver fatigue," in Proc. 1997 IEEE Conf. Intelligent Transportation System, Nov. 1997, pp. 314-319.

[11] R. Grace, A. Guzman, J. Staszewski, B. A. Peters, M. Mallis, and D. F. Dinges, "The Carnegie Mellon TruckSim: A tool to improve driving safety," in Proc. 1998 IEEE 17th Conf. Digital Avionics Systems, vol. 2, Nov. 1998, pp. I35/1-I35/831.

[12] R. Grace, V. E. Byrne, D. M. Bierman, J. M. Legrand, D. Gricourt, B. K. Davis, J. J. Staszewski, and B. Carnahan, "A drowsy driver detection system for heavy vehicles," in Proc. 17th AIAA/IEEE/SAE Conf. Digital Avionics Systems, vol. 2, Nov. 1998, pp. I36/1-I36/8.

[13] C. A. Perez, A. Palma, C. A. Holzmann, and C. Pena, "Face and eye tracking algorithm based on digital image processing," in Proc. IEEE Int. Conf. Systems, Man, Cybernetics, vol. 2, Oct. 2001, pp. 1178-1183.

[14] T. Pilutti and G. Ulsoy, "Identification of driver state for lane-keeping tasks," IEEE Trans. Syst, Man, Cybern. A, Syst. Humans, vol. 29, pp. 486-502, Sep. 1999.

[15] J. C. Popieul, P. Simon, and P. Loslever, "Using driver's head movements evolution as a drowsiness indicator," in Proc. IEEE Int. Intelligent Vehicles Symp., Jun. 2003, pp. 616-621.

[16] J. Qiang, Z. Zhiwei, and P. Lan, "Real-time nonintrusive monitoring and prediction of driver fatigue," IEEE Trans. Veh. Technol., vol. 53, pp. 1052-068, Jul. 2004

[17] R. S. Huang, C. J. Kuo, L. L. Tsai, and O. T. C. Chen, "EEG pattern recognition-arousal states detection and classification," in Proc. IEEE Conf. Neural Networks, vol. 2, Jun. 1996, pp. 641-646.

[18] A. Vuckovic, V. Radivojevic, A. C. N. Chen, and D. Popovic, "Automatic recognition of alertness and drowsiness from EEG by an artificial neural network," Med. Eng. Phys., vol. 24, pp. 349-360, Jun. 2002.

[19] S. Roberts, I. Rezek, R. Everson, H. Stone, S. Wilson, and C. Alford, "Automated assessment of vigilance using committees of radial basis function analyzers," Inst. Elect. Eng. Science Measure. Technol., vol. 147, pp. 333-338, Nov. 2000.

[20] K. B. Khalifa, M. H. Bedoui, R. Raytchev, and M. Dogui, "A portable device for alertness detection," in Proc. 1st Ann. Int. IEEE-EMBS Special Topic Conf. Microtechnologies in Medicine and Biology, Oct. 2000, pp. 584-586.

[21] B. J. Wilson and T. D. Bracewell, "Alertness monitor using neural networks for EEG analysis," in Proc. IEEE Signal Processing Society Workshop on Neural Networks for Signal Processing X, vol. 2, Dec. 2000, pp. 814-820.

[22] J. A. Stern, D. Boyer, and D. Schroeder, "Blink rate: Possible measure of fatigue," Human Factors, vol. 36, pp. 285-297, 1994.

[23] J. A. Stern, L. C. Walrath, and R. Goldstein, "The endogenous eyeblink," Psychophysiol., vol. 21, pp. 22-33, 1984.

[24] D. Schmidt, L. A. Abel, L. F. Dell'Osso, and R. B. Daroff, "Saccade velocity characteristics: Intrinsic variability and fatigue," Aviation Space Environ. Med., vol. 50, pp. 393-395, 1979.
[25] D. K. McGregor and J. A. Stern, "Time on task and blink effects on saccade duration," Ergonomics, vol. 39, pp. 649-660, 1996.

[26] J. Santamaria and K. Chiappa, "The EEG of drowsiness in normal adults," J. Clin. Neurophysiol., vol. 4, no. 4, pp. 327-382, 1987.

[27] A. Rechtschaffen and A. Kales, Eds., "A manual of standardized terminology. in techniques and scoring system for sleep stages of human subjects," in BIS/BRI. Los Angeles, CA: UCLA, 1968.

[28] R. B. Berry, Sleep Medicine Pearls, S. A. Sahn and J. E. Heffner, Eds. Philadelphia, PA: Hanley and Belfus, 1999.

[29] P. Parikh and E. Micheli-Tzanakou, "Detecting drowsiness while driving using wavelet transform," in Proc. IEEE 30th Annual Northeast on Bioengineering Conf., Apr. 2004, pp. 79-80.

[30] H. Park, "Automated sleep stage analysis using hybrid rule-based and case-based reasoning," Ph.D. dissertation, Seoul National Univ., Korea, 2000.

[31] T. P. Jung, S. Makeig, M. Stensmo, and T. J. Sejnowski, "Estimating alertness from the EEG power spectrum," IEEE Trans. Biomed. Eng., vol. 44, pp. 60-69, 1997.

[32] S. Makeig and T. P. Jung, "Tonic, phasic and transient EEG correlates of auditory awareness in drowsiness," Cogn. Brain Res., vol. 4, pp. 15-25, 1996.

[33] K. Van Orden, W. Limbert, S. Makeig, and T. P. Jung, "Eye activity correlates of workload during a visualspatial memory task," Human Factors, vol. 43, no. 1, pp. 111-21, 2001.

[34] S. Makeig and M. Inlow, "Lapses in alertness: Coherence of fluctuations in performance and EEG spectrum," Electroencephalogr. Clin. Neurophysiol., vol. 86, pp. 23-35, 1993.

[35] M. Treisman, "Temporal rhythms and cerebral rhythms," in Timing and Time Perception, J. Gibbon and L. Allan, Eds. New York: Academic, 1984, vol. 423, pp. 542-565.

[36] J. Beatty, A. Greenberg, W. P. Deibler, and J. O'Hanlon, "Operant control of occipital theta rhythm affects performance, in a radar monitoring task," Science, vol. 183, pp. 871-873, 1974.

[37] C. Jutten and J. Herault, "Blind separation of sources I. An adaptive algorithm based on neuromimetic architecture," Signal Process., vol. 24, pp. 1-10, 1991.

[38] J. F. Cardoso and A. Souloumiac, "Blind beamforming for non Gaussian signals," IEEE Proc. F 140, vol. 6, pp. 362-370, 1993.

[39] P. Comon, "Independent component analysis - A new concept?," Signal Process., vol. 36, pp. 287-314, 1994.

[40] A. J. Bell and T. J. Sejnowski, "An information-maximization approach to blind separation and blind deconvolution," Neural Computat., vol. 7, pp. 1129-1159, 1995.

[41] J. F. Cardoso and B. Laheld, "Equivariant adaptive source separation," IEEE Trans. Signal Process., vol. 45, pp. 434-444, 1996.

[42] D. T. Pham, "Blind separation of instantaneous mixture of sources via an independent component analysis," IEEE Trans. Signal Process., vol. 44, pp. 2768-2779, 1997.

[43] M. Girolami, "An alternative perspective on adaptive independent component analysis," Neural Computat., vol. 10, pp. 2103-2114, 1998.

[44] T. W. Lee, M. Girolami, and T. J. Sejnowski, "Independent component analysis using an extended infomax algorithm for mixed sub-Gaussian and super-Gaussian sources," Neural Computat., vol. 11, pp. 606-633, 1999.

[45] S. Makeig, A. J. Bell, T. P. Jung, and T. J. Sejnowski, "Independent component analysis of electroencephalographic data," Advances in Neural Information Process. Syst., vol. 8, pp. 145-151, 1996.

[46] T. P. Jung, C. Humphries, T. W. Lee, S. Makeig, M. J. McKeown, V. Iragui, and T. J. Sejnowski, "Extended ICA removes artifacts from electroencephalographic recordings," Advances in Neural Information Process. Syst., vol. 10, pp. 894-900, 1998.

[47] T. P. Jung, S. Makeig, C. Humphries, T. W. Lee, M. J. McKeown, V. Iragui, and T. J. Sejnowski, "Removing electroencephalographic artifacts by blind source separation," Psychophysiol., vol. 37, pp. 163-78, 2000.

[48] T. P. Jung, S. Makeig, W. Westerfield, J. Townsend, E. Courchesne, and T. J. Sejnowski, "Analysis and visulization of single-trial event-related potentials," Human Brain Mapping, vol. 14, no. 3, pp. 166-85, 2001

[49] A. Yamazaki, T. Tajima, and K. Matsuoka, "Convolutive independent component analysis of EEG data," in Ann. Conf. SICE, vol. 2, Aug. 2003, pp. $1227-1231$.

[50] A. Meyer-Base, D. Auer, and A. Wismueller, "Topographic independent component analysis for fMRI signal detection," in Proc. Int. Joint Conf. Neural Networks, vol. 1, Jul. 2003, pp. 601-605. 
[51] M. Naganawa, Y. Kimura, K. Ishii, K. Oda, K. Ishiwata, and A. Matani, "Extraction of a plasma time-activity curve from dynamic brain pet images based on independent component analysis," IEEE Trans. Biomed. Eng., vol. 52, pp. 201-210, Feb. 2005.

[52] R. Liao, J. L. Krolik, and M. J. McKeown, "An information-theoretic criterion for intrasubject alignment of FMRI time series: Motion corrected independent component analysis," IEEE Trans. Med. Imag., vol. 24, pp. 29-44, Jan. 2005.

[53] WorldToolKit Reference Manual, 7 ed., SENSE8 Corp., Mill Valley, 1997.

[54] A. Papoulis, "Minimum bias windows for high resolution spectral estimation," IEEE Trans. Info. Theory, vol. IT-19, pp. 9-12, 1973.

[55] M. Steriade, "Central core modulation of spontaneous oscillations and sensory transmission in thalamocortical systems," Current Opin. Neurobiol., vol. 3, no. 4, pp. 619-625, 1993.

[56] A. Destexhe, D. Contreras, and M. Steriade, "Spatiotemporal analysis of local field potentials and unit discharges in cat cerebral cortex during natural wake and sleep states," J. Neurosci., vol. 19, pp. 4595-4608, 1999.

[57] P. Achermann and A. A. Borbely, "Low frequency oscillations in the human sleep electroencephalogram," Neurosci., vol. 81, pp. 213-222, 1997.

[58] E. Werth, P. Achermann, D. J. Dijk, and A. A. Borbely, "Spindle frequency activity in the sleep EEG: Individual differences and topographic distribution," Electroencephalogr. Clin. Neurophysiol., vol. 103, pp. 535-542, 1997.

[59] D. Gervasoni, S. C. Lin, S. Ribeiro, E. S. Soares, J. Pantoja, and M. A. L. Nicolelis, "Global forebrain dynamics predict rat behavioral states and their transitions," Journal of Neuroscience, vol. 24, pp. 11 137-11 147, Dec. 2004.

[60] S. Chatterjee and A. S. Hadi, "Influential observations, high leverage points, and outliers in linear regression," Statist. Sci., pp. 379-416, 1986.

[61] N. R. Draper and H. Smith, Applied Regression Analysis New York, 1998, Wiley Series in Probability and Statistics.

[62] C. T. Lin, R. C. Wu, T. P. Jung, S. F. Liang, and T. Y. Huang, "Estimating driving performance based on EEG spectrum analysis," EURASIP $J$. Appl. Signal Process., to be published.
Dr. Lin was elected an IEEE Fellow for his contributions to biologically inspired information systems. He serves on the Board of Governors of the IEEE Circuits and Systems (CAS) Society (2005) and the IEEE Systems, Man, Cybernetics (SMC) Society during 2003-2005. He was the Distinguished Lecturer of the IEEE CAS Society from 2003 to 2005. He is the International Liaison of International Symposium of Circuits and Systems (ISCAS) 2005 in Japan, the Special Session Co-Chair of ISCAS 2006 in Greece, and the Program Co-Chair of IEEE International Conference on SMC 2006 in Taiwan, R.O.C. He has been the President of Asia Pacific Neural Network Assembly since 2004. He has received the Outstanding Research Award granted by National Science Council, Taiwan, R.O.C., since 1997 to present, the Outstanding Electrical Engineering Professor Award granted by the Chinese Institute of Electrical Engineering (CIEE) in 1997, the Outstanding Engineering Professor Award granted by the Chinese Institute of Engineering (CIE) in 2000, and the 2002 Taiwan Outstanding Information-Technology Expert Award. He was also elected to be in the 38th Ten Outstanding Rising Stars in Taiwan, R.O.C (2000). He currently serves as an Associate Editor of the IEEE TRANSACTIONS ON CIRCUITS AND SYSTEMS- PART I AND II, the IEEE TRANSACTIONS ON Systems, MAN, and Cybernetics, the IEEE TRANSACTIONS ON FuZZY SYSTEMS, and the International Journal of Speech Technology. He is a member of the Tau Beta Pi, Eta Kappa Nu, and Phi Kappa Phi.

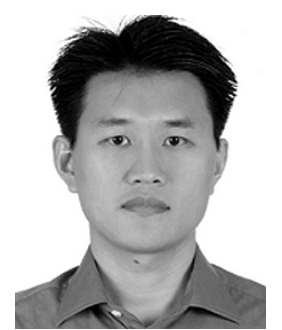

Ruei-Cheng Wu received the B.S. degree in nuclear engineering from National Tsing-Hua University, Taiwan, R.O.C., in 1995, and the M.S. and Ph.D. degrees in control engineering and electrical and control engineering from Nation Chiao-Tung University, Hsin-Chu, Taiwan, R.O.C., in 1997 and 2005, respectively.

He is currently with the Department of Electrical and Control Engineering, Nation Chiao-Tung University. His current research interests are biomedical signal processing, image processing, audio signal processing, fuzzy neural networks, and linear control.

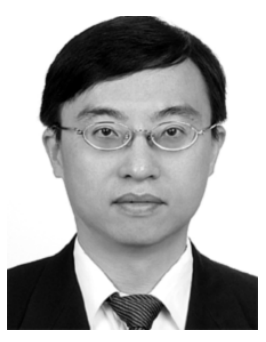

Chin-Teng (CT) Lin (F'05) received the B.S. degree from National Chiao-Tung University (NCTU), Hsin-Chu, Taiwan, R.O.C. in 1986 and the Ph.D. degree in electrical engineering from Purdue University, West Lafayette, IN, in 1992.

$\mathrm{He}$ is currently the Chair Professor of Electrical and Computer Engineering, Dean of Computer Science College, and Director of Brain Research Center, NCTU. He served as the Director of the Research and Development Office of NCTU from 1998 to 2000, the Chairman of Electrical and Control Engineering Department of NCTU from 2000 to 2003, and Associate Dean of the College of Electrical Engineering and Computer Science from 2003 to 2005. His current research interests are fuzzy neural networks, neural networks, fuzzy systems, cellular neural networks, neural engineering, algorithms and VLSI design for pattern recognition, intelligent control, and multimedia (including image/video and speech/audio) signal processing, and intelligent transportation system (ITS). He is the coauthor of Neural Fuzzy Systems- A Neuro-Fuzzy Synergism to Intelligent Systems (Englewood Cliffs, NJ: Prentice-Hall), and the author of Neural Fuzzy Control Systems with Structure and Parameter Learning (Singapore: World Scientific). He has published more than 90 journal papers in the areas of neural networks, fuzzy systems, multimedia hardware/software, and soft computing, including approximately 60 IEEE journal papers.

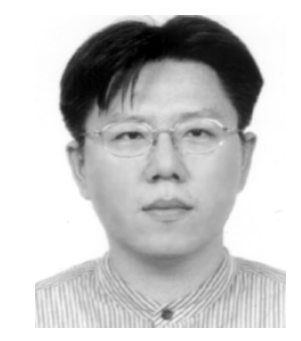

Sheng-Fu Liang was born in Tainan, Taiwan, in 1971. He received the B.S. and M.S. degrees in control engineering from the National Chiao-Tung University (NCTU), Hsin-Chu, Taiwan, R.O.C., in 1994 and 1996, respectively. He received the Ph.D. degree in electrical and control engineering from NCTU in 2000

From 2001 to 2005, he was a Research Assistant Professor with the Department of Electrical and Control Engineering, NCTU. In 2005, he joined the Department of Biological Science and Technology, NCTU, where he serves as an Assistant Professor. He has also served as the chief executive of Brain Research Center, NCTU Branch, University System of Taiwan, R.O.C., since September 2003. His current research interests are biomedical engineering, biomedical signal/image processing, machine learning, fuzzy neural networks (FNN), the development of brain-computer interface $(\mathrm{BCI})$, and multimedia signal processing.

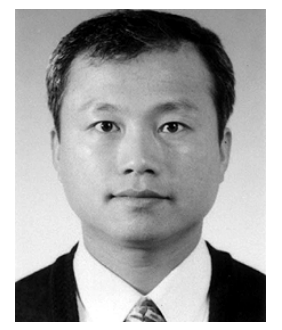

Wen-Hung Chao received the M.S. degree in biomedical engineering from National Cheng Kung University, Tainan, Taiwan, R.O.C., in 1996.

$\mathrm{He}$ is currently pursuing the Ph.D. degree with the Department of Electrical and Control Engineering, National Chiao-Tung University, Hsin-Chu, Taiwan, R.O.C. Since August 1996, he has been a Lecturer with the Department of Biomedical Engineering, Yuanpei University of Science and Technology, Hsin-Chu, Taiwan, R.O.C. His current research interests are biomedical signal processing, fuzzy neural networks, and designing biomedical instruments. 


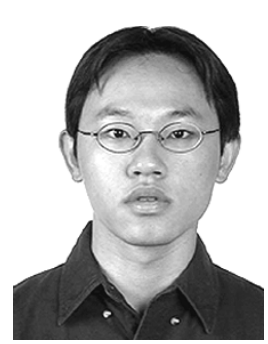

Yu-Jie Chen received the B.S. and M.S. degrees, both in electrical and control engineering, from National Chiao-Tung University, Hsin-Chu, Taiwan, R.O.C., in 2003 and 2005, respectively.

$\mathrm{He}$ is currently with the Department of Electrical and Control Engineering, National Chiao-Tung University. His research interests are in the areas of digital signal processing, biomedical signal processing, and fuzzy neural networks.

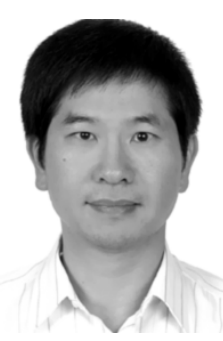

Tzyy-Ping Jung received the B.S. degree in electronics engineering from National Chiao Tung University, Hsin-Chu, Taiwan, R.O.C., in 1984, and the M.S. and Ph.D. degrees in electrical engineering from The Ohio State University, in 1989 and 1993, respectively.

He was a Research Associate with the National Research Council of the National Academy of Sciences and with the Computational Neurobiology Laboratory, The Salk Institute, San Diego, CA. $\mathrm{He}$ is currently an Associate Research Professor with the Institute for Neural Computation of University of California, San Diego (UCSD). He is also the Associate Director of the Swartz Center for Computational Neuroscience at UCSD. His research interests are in the areas of biomedical signal processing, cognitive neuroscience, artificial neural networks, time-frequency analysis of human EEG, functional neuroimaging, and the development of neural human-system interfaces. 